\title{
ITEM C
}

Livros, artigos de divulgação científica, relatórios técnicos 
001 AMARAL, G. (1977) Application of Landsat imagery to regional geological mapping of the Brazilian Amazon Region. Rel. INPE 1010-NTE/080, p. 5-6.

002 AMARAL, G. (1977) Automatic interpretation of Landsat imagery for prospection of mineral deposits in the Amazon region. Rel. INPE 1010-NTE/080, p. 7-9.

003 AMARAL, G.; MATTOS, J. T.; ALMEIDA FILHO, R.; CUNHA, R. P.; PARADELLA, W. R. (1977) Application of Landsat imagery to regional geological mapping the Belo Horizonte Quadrangle. Rel. INPE 1010-NTE/080, p. 13-15.

004 CORDANI, U. G.; BARBOSA, G. V.; LOURENÇO, J. S.; FIGUEIREDO FILHO, P. M.; SILVA, R. P. (1977) Avaliação e perspectivas: área de Geociências. CNPq, 39p.

005 FERNANDES, P. E. C. A.; PINHEIRO, S. S.; MONTALVÃO, R. M. G.; ISSLER, R. S.; ABREU, A. S.; TASSINARI, C. C. G. (1977) Levantamento de Recursos Naturais, v. 14, Folha SA-19 IçaRio de Janeiro, Projeto RADAMBRASIL-DNPM, p. 21-123.

006 GOMES, C. B. (1977) Meteoritos: um tema atual de pesquisa. O Estado de São Paulo, Suplemento Cultural, 20: 14-15, 27 fev.

007 GOMES, C. B. (1977) Tectitos: um material de natureza extraterrestre? O Estado de São Paulo, Suplemento Cultural, 41: 6-7, 24 jul.

008 MENDES, J. C. (1977) Ação universitária e comunidade. O Estado de São Paulo, Suplemento Cultural, 53: 16,16 out.

009 MENDES, J. C. (1977) Paleontologia Geral. São Paulo, Livros Técnicos Científicos, EDUSP, 342p.

010 MENDES, J. C. (1977) Vida pré-histórica. Rev. Prisma Brasileira no 38, Ed. Melhoramentos, Inst. Nac. Livro, EDUSP, 160p.

011 PETRI, S. (1977) Evolução da atmosfera. O Estado de São Paulo, Suplemento Cultural, 29: 11-12, 01 maio.

012 PIMENTA, O. N. S. \& TASSINARI, C. C. G. (1977) Reconhecimento geológico dos Rios Telles Pires, Apiacá e Ximari, nos Estados do Amazonas e Mato Grosso. Projeto RADAMBRASIL, Relatório Interno, 19p.

013 PIMENTA, O. N. S. \& TEIXEIRA, W. (1977) Campanha dos Rios Juruena, Peixe e Arinos, MTJulho/Agosto. Projeto RADAMBRASIL, Relatório Interno, 19p.

014 REBOUÇAS, A. C. (1977) O infindável ciclo da água. O Estado de São Paulo, Suplemento Cultural, 20: $10-11,27 \mathrm{fev}$.

015 RIBEIRO FILHO, E. (1977) Minérios e balança comercial. O Estado de São Paulo, Suplemento Cultural, 34: 14-15, 05 jun.

016 RIBEIRO FILHO, E. (1977) A importância dos bens minerais. O Estado de São Paulo, Suplemento Cultural, 60: 16, $04 \mathrm{dez}$.

017 ROCHA CAMPOS, A. C. (1977) Dinossauros: fatos e mitos. O Estado de São Paulo, Suplemento Cultural, 50: 13-14, 25 set.

018 RÖSLER, O. (1977) Antigas florestas da Antártida. O Estado de São Paulo, Suplemento Cultural, 46: 3-4, 28 ago.

019 RÖSLER, O. (1977) Programa e endereços. I Reunião de Paleobotânicos e Palinólogos, Instituto de Geociências, USP, São Paulo, SP, p. 1-15.

020 RƯEGG, N. R.; RÖSLER, O.; REBOUÇAS, A. C.; SADOWSKI, G. R. (1977) Índice bibliográfico 1975-1976, Instituto de Geociências, USP, São Paulo, SP. Bol. IG-USP, 8: 139-167. Coordenadores Editoriais.

021 SANTOS, A. R.; CHANG, L. C.; PARADELLA, W. R.; MENEZES, P. R.; BARBOSA, M. P.; AMARAL, G.; CREPANI, E. (1977) Projeto mapa geológico ao milionésimo. Rel. INPE 1074NTE/100. 
SUGUIO, K. (1977) O Homem e o oceano. [Tradução e adaptação do original de B. J. Skinner e K. K. Turekian] São Paulo, Ed. Edgard Blücher, EDUSP, 136p.

SUGUIO, K. (1977) Formação costeira e implicações humanas. O Estado de São Paulo, Suplemento Cultural, 18: 13, 13 fev.

024 SUGUIO, K. (1977) Flutuações do nivel do mar. O Estado de São Paulo, Suplemento Cultural, 35 : 4-5, 12 jun.

025 SUGUIO, K. (1977) Annotated bibliography on Quaternary shorelines and sea-level changes in Brazil. Contr. PICG-União Internacional de Ciências Geológicas, 35p.

026 TASSINARI, C. C. G. \& TEIXEIRA, W. (1977) Geocronologia da Folha SB-20 Purus-Belém. Projeto RADAMBRASIL, Relatório Interno, (RIR 137G), 15p.

027 TEIXEIRA, E. (1977) Levantamento de Recursos Naturais, v. 13 - Folhas SB/SC-18 JavariContamana. Projeto RADAMBRASIL-DNPM, p. 1-103.

028 TEIXEIRA, W. (1977) Levantamento de Recursos Naturais, v. 14 - Folha SA-19 Iça. Projeto RADAMBRASIL-DNPM, p. 1-87.

029 TEIXEIRA, W. \& TASSINARI, C. C. G. (1977) Estudo geocronológico Rb-Sr e K-Ar da Folha SC-20 Porto Velho. Projeto RADAMBRASIL, Relatório Interno, (RIR 127G), 34p.

030 TEIXEIRA, W. \& TASSINARI, C. C. G. (1977) Estudo geocronológico da Folha SA-20 ManausBelém. Projeto RADAMBRASIL, Relatório Interno (RIR 145G), 16p.

031 VALARELLI, J. V. (1977) Geologia da área mineralizada, Membro Azul, Manganês. Rel. Amazônia Mineração. 54p.

032 VALARELLI, J. V. (1977) Cristalografia geométrica bidimensional. Ed. Preliminar (off-set). 50p.

1978

033 BATISTA, L. F. \& REBOUÇAS, A. C. (1978) Ação programada em ciência e tecnologia 20 - Recursos Naturais, Hídricos. Publ. Esp. SEPLAN-CNPq, p. 105-179.

034 CARVALHO, A.; QUEIROZ NETO, J. P.; JOURNAUX, A. (1978) Carta Geomorfológica do Vale do Rio do Peixe em Marília, SP. Bol. Inst. Geogr., USP, 10, 23p. Coordenadores Editoriais.

035 CARVALHO, A.; QUEIROZ NETO, J. P.; JOURNAUX, A. (1978) Carta de Formaçб̃es superficiais do Vale do Rio do Peixe em Marília, SP. Bol. Inst. Geogr., USP, 11, 18p. Coordenadores Editoriais.

036 CORDANI, U. G. (1978) Comentários sobre as determinações geocronológicas disponíveis na Folha Paranapanema. Carta Geológica do Brasil ao Milionésimo, DNPM, Folha Paranapanema, SF-22, p. 43-45.

037 CORDANI, U. G. \& BRITO NEVES, B. B. (1978) Geocronologia do pré-cambriano. In: H. A. V. Inda J. F. Barbosa. Texto explicativo para o Mapa Geológico da Bahia, CPM-SME, Bahia, I parte, cap. 5: 32-49.

038

GOMES, C. B. (1978) Ocorrências de meteoritos no Brasil. O Estado de São Paulo, Suplemento Cultural, 99: 10-11, 24 set.

039 LEAL, J. W. L.; SILVA, G. H.; SANTOS, D. B.; TEIXEIRA, W.; LIMA, M. I. C.; FERNANDES, C. A. C.; PINTO, A. C. (1978) Levantamento dos Recursos Naturais, v. 15. - Geologia, Folha SC20 Porto Velho-Rio de Janeiro. Projeto RADAMBRASIL-DNPM, p. 1-184.

040 LOURENÇO, R. S.; MONTALVÃO, R. M.; PINHEIRO, S. S.; FERNANDES, C. A. C.; TEIXEIRA, W. (1978) Levantamento de Recursos Naturais, v. 18 - Geologia, Folha SA-20 Manaus-Rio de Janeiro. Projeto RADAMBRASIL-DNPM, p. 1-164.

041 MENDES, J. C. (1978) A pesquisa paleontológica no Brasil. O Estado de São Paulo, Suplemento Cultural, 89: 11, 09 jul.

042 MENDES, J. C. (1978) Universidade de São Paulo: súmula de sua história. Publ. ACIESP, 48p.

043 MENDES, J. C. (1978) A pós-graduação no Brasil. O Estado de São Paulo, Suplemento Cultural, 101: 8-9, 08 out. 
044 MENDES, J. C. (1978) Belezas naturais criadas pela erosão. O Estado de São Paulo, Suplemento Cultural, 103: 10, 22 out.

045 PETRI, S. (1978) Tempo geológico. O Estado de São Paulo, Suplemento Cultural, 92: 13-14, 30 jul.

046 PETRI, S.; SZAJNBOK, M.; CAROLI, A. J. (1978) Reflexos do ensino de 20 grau no exames vestibulares da FUVEST. Simpósio sobre o Ensino Biológico, Física, Matemática e Química (10 e 2! graus) no Estado de São Paulo, São Paulo, SP, ACIESP, p. 25-43.

047 REBOUÇAS, A. C. (1978) Água: problemática nordestina. O Estado de São Paulo, Suplemento Cultural, 76: 4-16,02 abr.

048 REBOUÇAS, A. C. (1978) O grande potencial hidrotermomineral do Brasil. O Estado de São Paulo, Suplemento Cultural, 72: 8-12, 05 mar.

049 REBOUÇAS, A. C. (1978) Na seca do sul não falta água. O Estado de São Paulo, Suplemento Cultural, $71: 5-8,18$ jun.

050 REBOUÇAS, A. C. (1978) Águas Subterrâneas no Brasil - Projeto 03. Recursos Hídricos, CNPq. p. 46-54.

051 RIBEIRO FILHO, E. (1978) Energia dos combustiveis fósseis. O Estado de São Paulo, Suplemento Cultural, 108: 3-4, 26 nov.

052 RÖSLER, O. (1978) Advances in Paleobotany and Allied Sciences in Brazil. Bol. IG-USP, 9: 57152. Coordenador Editorial.

053 TASSINARI, C. C. G. \& TEIXEIRA, W. (1978) Estudo geocronológico das rochas pré-cambrianas da Folha SC-21 - Juruena-Salvador. Projeto RADAMBRASIL, Relatório Interno, (RIR 168G), $23 p$.

054 TASSINARI, C. C. G.; TEIXEIRA, W.; SIGA Jr., O. (1978) Estudo geocronológico Rb-Sr e K-Ar da Folha SD-20 Guaporé-Goiânia. Projeto RADAMBRASIL, Relatório Interno, (RIR 178G), $11 \mathrm{p}$.

055 TEIXEIRA, W. (1978) Levantamento de Recursos Naturais, v. 17, Folha SB-20 Purus-Rio de Janeiro. Projeto RADAMBRASIL-DNPM, p. 1-128.

056 TEIXEIRA, W.; TASSINARI, C. C. G.; SIGA Jr., O. (1978) Interpretação geocronológica da Folha SA-24 Fortaleza-Natal. Projeto RADAMBRASIL, Relatório Interno, 18p.

1979

057 AMARAL, G.; CHIANG, L. C.; ALMEIDA FILHO, R. (1979) Mapa geológico de parte do Estado de São Paulo baseado em imagens Landsat. Rel. INPE 1437-RPE/012, 20p.

ARCHANGELSKY, S.; GAMERO, J. C.; RÖSLER, O. (1979) Bibliografia Paleobotânica y Palinológica Latinoamericana 1978-1979. Bol. Ass. Latinoam. Paleobot. Palin., 6: 11-22.

059 BECK, M. T. T.; KARMANN, I.; SANCHEZ, L. E. (1979) Espeleologia: ciência e esporte. O Estado de São Paulo, Suplemento Cultural, 118: 15-16, 04 fev.

060 CORDANI, U. G. \& TEIXEIRA, W. (1979) Comentários sobre determinações geocronológicas disponiveis na Folha do Rio de Janeiro. Carta Geológica do Brasil ao Milionésimo, DNPM, Folha Rio de Janeiro, SF-23, p. 175-207.

061 FÚlfFARO, V. J. \& SUGUIO, K. (1979) Vertical movements in continental southern Brazil during the Cenozoic. In: Earth Rheology, Isostasy and Eustasy (N. A. Mörner, ed.), Inglaterra, John Wiley and Sons. Ltd. Publ., p. 419-425.

062 GOMES, C. B. (1979) O lixo atômico. O Estado de São Paulo, Suplemento Cultural, 136: 14, 10 jun.

063 GOMES, C. B. (1979) Programa nuclear brasileiro: uma análise crítica. O Estado de São Paulo, Suplemento Cultural, 139: 12-13, 01 jul.

064 GOMES, C. B. (1979) A disposição do lixo atômico: aspectos geológicos. O Estado de São Paulo, Suplemento Cultural, 144: 11-12, 05 ago. 

145: 3-4, 12 ago.

066 KIHARA, Y.; BATTAGIN, A. F.; MARCIANO Jr., E.; SHUKUZAWA, J. K. (1979) Contribuição ao controle industrial de qualidade de cimento portland brasileiro através da técnica de espectrometria de raios X. XXIX Reunião de técnicos da indústria de cimento, ABCP, São Paulo, SP, Anexo 6, 69p.

067 LOURENÇO, R. S.; MONTALVÃO, R. M. G.; PINHEIRO, S. S.; PEREIRA, E. R.; FERNANDES, C. A. C.; TEIXEIRA, W. (1979) Levantamento dos Recursos Naturais, v. 18 - Folha SA-20 Manaus. Projeto RADAMBRASIL, 164p.

068 NASCIMENTO, D.; TEIXEIRA, W.; BARROS, M. J. G. (1979) Levantamento dos Recursos Naturais - Geologia, Folha SA-24 Fortaleza. Projeto RADAMBRASIL-DNPM, p. 1-212.

069 PETRI, S. (1979) Subsídios da geologia à história e à pré-história. O Estado de São Paulo, Suplemento Cultural, 12:11-13, 05 ago.

070 RIBEIRO FILHO, E. (1979) O homem e o ambiente geológico. O Estado de São Paulo, Suplemento Cultural, 136: 15-16, 10 jun.

071 RIBEIRO FILHO, E. (1979) Recursos minerais da Amazônia. Associação Nacional de Programação Econômica e Social, ANPES, São Paulo, SP, 23p.

072 RÖSLER, O. (1979) O carvão no mundo e as pesquisas sobre os Sistemas Carbonífero e Permiano. Jornal do Geólogo, 7: 16.

073 RÖSLER, O. (1979) A Associação Latinoamericana de Paleobotânica e Palinologia, ALPP. Jornal do Geólogo, 7: 16.

074 RÖSLER, O. (1979) II Reunião de Paleobotânicos e Palinólogos, volume de programa e resumos. Paleobot. Latinoam., Circ. ALPP, 1(3): 17-35. Coordenador Editorial.

075 RÖSLER, O. (1979) Notícias sobre Paleobotânica e Palinologia na América Latina. Paleobot. Latinoam., Circ. ALPP. 1(3): 1-6.

076 RÖSLER, O. (1979) Paleobotânicos e Palinólogos da América Latina. Paleobot. Latinoam., Circ. ALPP, 1(2): 1-10.

077 RÖSLER, O. (1979) IX-ICC: um congresso paleobotânico. Bol. Ass. Latinoam. Paleobot. Palin., 6: $23-24$.

078 RÖSLER, O. (1979) Prioridades na pesquisa geológica do Estado de São Paulo. II Simp. Geol. Reg., Rio Claro, SP, 2: 353-358. (Relator)

079 SANTOS, R. O. B.; PITHAN, J. H. L.; BARBOSA, E. S.; FERNANDES, C. A. C.; TASSINARI, C. C. G.; CAMPOS, D. A. (1979) Levantamento de Recursos Naturais, Folha SD-20 GuaporéRio de Janeiro, Projeto RADAMBRASIL-DNPM, 92p.

080 SUGUIO, K. (1979) O passado remoto: métodos de datação. O Estado de São Paulo, Suplemento Cultural, 149: 6-7, 09 set.

081 SUGUIO, K. \& BIGARELLA, J. J. (1979) Ambiente Fluvial. In: Ambientes de sedimentação, sua interpretação e importância (J. J. Biagrella, K. Suguio e R. D. Becker, eds.) Curitiba, PR, Ed. Univ. Fed. Paraná/Associação de Defesa e Educação Ambiental, 183p.

082 SUGUIO, K.; FAIRCHILD, T. R.; MARTIN, L.; FLEXOR, J. M. (1979) International Symposium . on Coastal Evolution in the Quaternary, São Paulo, Brazil. Seção de Ilustrações Técnicas do IAG-USP, Proceedings, 606p. Coordenadores Editorais.

083 SUGUIO, K.; MARTIN, L.; FLEXOR, J. M. (1979) Sea level fluctuations during the past 6,000 years along the State of São Paulo, Brazil. In: Earth Rheology, Isostasy and Eustasy (N. A. Mörner, ed.), John Wiley and Sons. Ltd. Publ., Inglaterra, p. 471-486.

084 SVISERO, D. P.; MEYER, H. O. A.; TSAI, H. M. (1979) Kimberlites in Brazil: an initial report. In: Kimberlites, diatremes and diamonds: their geology, petrology and geochemistry. Washington, USA, Ed. F. R. Boyd and H. O. A. Meyer. p. 92-100.

085 TEIXEIRA, W. (1979) Avaliação preliminar do acervo geocronológico das Folhas SG-21 Assunción, SG-22 Curitiba e SG-23 Iguape. Projeto RADAMBRASIL, Relatório Interno, (RIR 245G), 32p.

086 VALARELLI, J. V. (1979) Processo de obtenção de produto utilizável diretamente no solo, como fonte de potássio, ou correlatos. Patente de Invenção, IPT, Inst. Nac. Prop. Ind. nọ 7905941, 7p. 
087 AMARAL, G.; MATTOS, J. T.; ALMEIDA FILHO, R.; CUNHA, R. P.; PARADELLA, W. R. (1980) Carta geológica ao milionésimo da Folha Belo Horizonte com base na interpretação de imagens Landsat. Rel. INPE 1899-RPE/239, 27p.

088 COUTINHO, J. M. V. (1980) Carta geológica da Região Metropolitana da Grande São Paulo. 1: 100.000 . Gov. Est. S. Paulo, Secr. Neg. Metrop. EMPLASA.

089 FÚlfaro, V. J.; GAMA, Jr., E. G.; SOARES, P. C. (1980) Revisão estratigráfica da Bacia do Paraná. PAULIPETRO, Consórcio CESP/IPT, BP-008/80, 155p.

090 GOMES, C. B. \& KEIL, K. (1980) Brazilian stone meteorites. Albuquerque, The University of New Mexico Press. 161p.

091 KIHARA, Y. \& BATTAGIN, A. F. (1980) Determinação do teor de escória em cimento. Simp. sobre normalização de cimento, concreto e agregados, ABNT, São Paulo, SP, 17p.

092 KIHARA, Y.; BATTAGIN, A. F.; MARCIANO, Jr., E.; SHUKUZAWA, J. K. (1980) Aptidão à clinquerização de farinhas de cimento, fator de controle e qualidade do cimento. ABCP, São Paulo, SP, ET $-48,44 \mathrm{p}$.

093 LIMA, M. R. (1980) Bibliografia para o estudo do Cretáceo do Nordeste brasileiro. Craton e Intercraton, $5: 1-48$.

094 MENDES, J. C. (1980) As primeiras tentativas de produção de ferro no Brasil. O Estado de São Paulo, Suplemento Cultural, 168: $15-16,20$ jan.

095 MENDES, J. C. (1980) Lund, o pai da paleontologia brasileira. O Estado de São Paulo, Suplemento Cultural, 183: 14-15, 04 maio.

096 RÖSLER, O. (1980) Paleobotânica e áreas afins na América do Sul - introdução. Bol. IG-USP, 11: 31 .

097 RÖSLER, O. (1980) Atividades da ALPP (1978-1979) Paleobot. Latinoam., Circ. ALPP, 2 (1): $1-8$.

098 RÖSLER, O. (1980) Notícias sobre a Paleobotânica e Palinologia na América. Paleobot. Latinoam., Circ. ALPP, 2 (2): 1-15.

099 RÖSLER, O.\& FAIRCHILD, T. R. (1980) Paleobotânica e áreas afins na América do Sul. Bol. IG-USP, 11:31-189. Coordenadores Editoriais.

100 SUGUIO, K. (1980) Cobertura vegetal e preservação do meio físico terrestre.O Estado de São Paulo, Suplemento Cultural, 180: 12-13,13 abr.

101 SUGUIO, K. (1980) O homem do sambaqui. O Estado de São Paulo, Suplemento Cultural, 187: 9, 01 jun.

102 SUGUIO, K. (1980) Rochas sedimentares: propriedades, gênese e importância econômica. São Paulo, SP, Ed. Edgard Blücher Ltda./EDUSP, 500p.

103 SUGUIO, K. \& BARCELOS, J. H. (1980) Geologia Geral. Apostila para cursos de Biologia e Engenharia Agrícola, UNICAMP, Campinas. 181p.

104 TASSINARI, C. C. G. (1980) Estudo geocronológico da Folha ao milionésimo SD-22 Goiás-Goiânia. Projeto RADAMBRASIL, Relatório Interno, 58p.

105 TASSINARI, C. C. G. (1980) Comentários preliminares sobre a geocronologia da Folha SD-21, Cuiabá-Goiânia. Projeto RADAMBRASIL, Relatório Interno (RIR-293G), 13p.

106 TASSINARI, C. C. G. (1980) Estudo geocronológico da Folha SD-22 Tocantins-Goiânia. Projeto RADAMBRASIL, Relatório Interno (RIR 292G), 26 p.

107 TEIXEIRA, W. (1980) Folhas ao milionésimo SG-22 Porto Alegre, SH-21 Uruguaiana e SI-22 Lagoa Mirim: consideraçб̃es preliminares a respeito do quadro geocronológico existente. Projeto RADAMBRASIL, Relatório Interno, 31p.

108 TEIXEIRA, W. (1980) Acervo geocronológico das Folhas SB-24 Jaguaribe e SB-25 Natal: reavaliação e comentários afins. Projeto RADAMBRASIL, Relatório Interno, 38p.

109 TEIXEIRA, W. (1980) Levantamento de Recursos Naturais, v. 20, Folha SC-21 Juruena. Projeto RADAMBRASIL-DNPM, p. 1-116. 
110 CROWELL, J. C.; SUAREZ-SORUCO, R.; ROCHA CAMPOS, A. C. (1981) The Silurian Cancañiri (Zapla) Formation of Bolívia, Argentina and Peru. In: M. J. Hambrey and W. B. Harland (eds.), Earths's pre-Pleistocene glacial record, Cambridge Univ. Press, p. 902-907.

111 FITTIPALDI, F. C. \& RÖSLER, O. (1981) Preparação de cutículas paleozóicas. Paleobot. Latinoam., Circ. ALPP, 3 (4): 31.

112 GOMES, J. R. C.; GATTO, C. M. P.; SOUZA, G. M. C.; LUZ, D. S.; PIRES, J. L.; TEIXEIRA, W. (1981) Levantamento de Recursos Naturais, v. 23, Folha SB-24/25, Jaguaribe-Natal. Projeto RADAMBRASIL, p. $1-300$.

113 GUARDANI, R. \& VALARELLI, J. V. (1981) Processo de produção de termofosfato potássico. Patente de Invenção, IPT, Inst. Nac. Prop. Ind. n?818511, 10p.

114 MATSUE, K.; NUSKE, M. J.; BOSIO, N. J.; ELLERT, N.; SOUZA, I. M.; ROCHA CAMPOS, A. C.; ULBRICH, H. H. G. J.; AMARAL, G.; OLIVEIRA, M. A. F.; CARUSO, L. G.; CUNHA, C. A. L. (1981) Contribuição ao estudo de um currículo mínimo revisado para o Curso de Geologia. Simp. Nac. Ens. Geol. Brasil., Belo Horizonte, MG, Teses, 1: 1-9.

115 MENDES, J. C. (1981) A pesquisa paleontológica no Brasil. In: História das Ciências no Brasil, 3: 43-71, São Paulo, Ed. Pedagógica e Universitária, EDUSP.

116 NASCIMENTO, D. A.; GAVA, A.; PIRES, J. L.; TEIXEIRA, W. (1981) Levantamento de Recursos Naturais, v.21, Folha SA-24 Fortaleza, Projeto RADAMBRASIL, p.1-212.

117 ROCHA CAMPOS, A. C. (1981) Late Paleozoic "Série Tilitica" of Mozambique. In: M. J. Hambrey and W. B. Harland (eds.), Earth's pre-Pleistocene glacial record, Cambridge Univ. Press, p. 52-54.

118 ROCHA CAMPOS, A. C. (1981) Late Paleozoic tillites of the Sergipe-Alagoas Basin, Rondonia and Mato Grosso, Brazil. In: M. J. Hambrey and W. B. Harland (eds.), Earth's pre-Pleistocene glacial record, Cambdrige Univ. Press, p. 838-841.

119 ROCHA CAMPOS, A. C. (1981) Late Devonian Curuá Formation, Amazon Basin, Brazil. In: M. J. Hambrey and W. B. Harland (eds.), Earth's pre-Pleistocene glacial record, Cambridge Univ. Press, p. 888-891.

120 ROCHA CAMPOS, A. C. (1981) Middle-Late Devonian Cabeças Formation, Parnaíba Basin, Brazil. In: M. J. Hambrey and W. B. Harland (eds.), Earth's pre-Pleistocene glacial record, Cambridge Univ. Press, p. 892-895.

121 ROCHA CAMPOS, A. C. (1981) Late Ordovician (?) - early Silurian Trombetas Formation, Amazon Basin, Brazil. In: M. J. Hambrey and W. B. Harland (eds.), Earth's pre-Pleistocene glacial record, Cambridge Univ. Press, p. 896-898.

122 ROCHA CAMPOS, A. C. (1981) Early Palaeozoic Iapó Formation of Paraná, Brazil. In: M. J. Hambrey and W. B. Harland (eds.), Earth's pre-Pleistocene glacial record, Cambridge Univ. Press, p. 908-909.

123 ROCHA CAMPOS, A. C. (1981) The Cambrian (?) Limbo Group of Bolívia. In: M. J. Hambrey and W. B. Harland (ed.), Earth's pre-Pleistocene glacial record, Cambridge Univ. Press, p. 910-911.

124 ROCHA CAMPOS, A. C. (1981) The Late Precambrian Bebedouro Formation, Bahia, Brazil. In: M. J. Hambrey and W. B. Harland (eds.), Earth's pre-Pleistocene glacial record, Cambridge Univ. Press, p. 924-927.

125 ROCHA CAMPOS, A. C. (1981) Other Paleozoic and Precambrian conglomerate beds of uncertain origin in Brazil. In: M. J. Hambrey and W. B. Harland (eds.), Earth's pre-Pleistocene glacial record, Cambridge Univ. Press, p. 939-940.

126 ROCHA CAMPOS, A. C. \& HASUI, Y. (1981) Late Precambrian Jangada Group and Puga Formation of central western Brazil. In: M. J. Hambrey and W. B. Harland (eds.), Earth's pre-Pleistocene glacial record, Cambridge Univ. Press, p. 916-919.

127 ROCHA CAMPOS, A. C. \& HASUI, Y. (1981) Proterozoic diamictites of western Minas Gerais and eastern Goiás, central Brazil. In: M. J. Hambrey and W. B. Harland, (ed.), Earth's pre-Pleistocene glacial record, Cambridge Univ. Press, p. 920-923. 
ROCHA CAMPOS, A. C. \& HASUI, Y. (1981) Late Precambrian Salobro Formation of Brazil. In: M. J. Hambrey and W. B. Harland (eds.), Earth's pre-Pleistocene glacial record, Cambridge Univ. Press, p. 928-930.

129 ROCHA CAMPOS, A. C. \& HASUI, Y. (1981) Tillites of the Macaúbas Group (Proterozoic) in central Minas Gerais and southern Bahia, Brazil. In: M. J. Hambrey and W. B. Harland (eds.), Earth's pre-Pleistocene glacial record, Cambrige Univ. Press, p. 933-938.

130 ROCHA CAMPOS, A. C. \& SANTOS, P. R. (1981) Late Palaeozoic tillites of the Lutôe Series, Angola. In: M. J. Hambrey and W. B. Harland (eds.), Earth's pre-Pleistocene glacial record, Cambridge Univ. Press, p. 48-51.

131 ROCHA CAMPOS, A. C. \& SANTOS, P. R. (1981) The Itararé Subgroup, Aquidauana Group and San Gregório Formation, Paraná Basin, southeastern South America. In: M. J. Hambrey and W. B. Harland (eds.), Earth's pre- Pleistocene glacial record, Cambridge Univ. Press, p. 842-852.

132 RÖSLER, O. (1981) A III Reunião de Paleobotânicos e Palinólogos - notícias. Paleobot. Latinoam., Circ. ALPP, 3 (2): 1-7.

133 RÖSLER, O. (1981) A contribuição paleobotânica ao estudo do Carbonífero e Permiano no Brasil. Paleobot. Latinoam. Circ. ALPP, 3 (2): 23.

134 RÖSLER, O. (1981) III Reunião de Paleobotânicos e Palinólogos - volume informativo. Paleobot. Latinoam., Circ. ALPP, 3 (3): 7-14. Coordenador Editorial.

RŐSLER, O. (1981) Introdução ao volume de Resumos da III Reunião de Paleobotânicos e Palinólogos, São Paulo, SP, Paleobot. Latinoam., Circ. ALPP, 3 (4): 17.

136 SCHOBBENHAUS, C.; DERZE, G.R.; CAMPOS, D. A.; SANTOS, J. O. S.; SANTOS, E. J.; INDA, H. A. V.; HASUI, Y.; FONSECA, M. J. G.; MARINI, O. J.; DARDENE, M. A.; DANNI, J. C. M.; FUCK, R. A.; SANTOS, E. L.; RAMALHO, R.; LOGUÉRCIO, S. O. C.; SILVA, L. C. ORLANDI FILHO, V.; PONTE, F. C.; SCHORSCHER, H. D.; BRANDO, P. C. A.; MIGNON, R.; ASMUS, H. E.; GORINI, M. A.; PALMA, J. J. C.; CARVALHO, J. C.; AMARAL, C. A. B.; ALVES, E. C. (1981) Mapa geológico do Brasil e da Área Oceânica adjacente incluindo depósitos minerais, escala $1: 2.500 .000$, Ministério das Minas e Energia, Departamento Nacional da Produção Mineral, Brasilia.

137 TASSINARI, C. C. G. (1981) Comentários sobre a geocronologia da Folha SD-21 Cuiabá-Goiânia. Projeto RADAMBRASIL, Relatório Interno, 24p.

TASSINARI, C. C. G. (1981) Comentários geocronológicos sobre as rochas da Folha SF-21 Rio Apa-Goiânia. Projeto RADAMBRASIL, Relatório Interno, 24p.

TASSINARI, C. C. G. (1981) Estudo geocronológico da Folha SE-21 Corumbá-Goiânia. Projeto RADAMBRASIL, Relatório Interno. 15p.

140 TEIXEIRA, W. (1981) Folhas Aracajú e Recife, avaliação preliminar dos resultados radiométricos. Projeto RADAMBRASIL, Relatório Interno, 33p.

141 TEIXEIRA, W. (1981) Folhas SG-21 Assunción e SG-22 Curitiba, consideraçб̃es sobre os dados radiométricos: interpretação e evolução geocronológica. Projeto RADAMBRASIL, Relatório Interno, 41p.

142 ADAMS, C. J.; ARMSTRONG, R. L.; BAGDASARYAN, G. P.; BAKSI, A. K.; BALOGH, K.; BARNES, I. L.; BOELRIJK, N. A. I. M.; BONADONNA, F. P.; BONHOMME, M. G.; CASSIGNOL, C.; CHANIN, L.; GILLOT, P. Y.; GLEDHILL, A.; GOVINDARAJU, K.; HARAKAL, R.; HARRE, W.; HEBEDA, E. H.; HUNZIKER, J. C.; INGAMELLS, C. O.; KAWASHITA, K.; KISS, E.; KREUZER, H.; LONG, L. E.; MCDOWELL, F.; MEHNERT, H.; MONTIGNY, R.; PASTEELS, P.; RADICATI, F.; REX, D. C.; RUNDLE, C. C.; SAVELLI, C.; SONET, J.; WELIN, E.; ZIMMERMANN, J. L. (1982) Interlaboratory standards for dating purposes. In: Numerical dating in stratigraphy. Inglaterra, G. S. Odin, John Wiley \& Sons Ltda., p. 123-160. 
AMARAL, G. (1982) Unidades tectonoestratigráficas e problemas na classificação estratigráfica de rochas pré-cambrianas. Anexo ao Código e Guia de Nomenclatura Estratigráfica, Jornal do Geólogo, Supl. Esp., 17: 42-48.

AMARAL, G. (1982) Princípios básicos do sensoriamento remoto. Curso de aplicações geológicas do sensoriamento remoto para professores de aerofotogeologia. SBG-CNPq, p. 10-44.

AMARAL, G. \& MENEZES, P. R. (1982) Introdução ao curso de aplicações geológicas do sensoriamento remoto para professores de aerofotogeologia. SBG-CNPq, p. 1-9.

AMARAL, G. \& MENEZES, P. R. (1982) Radar de visada lateral. Curso de aplicações geológicas do sensoriamento remoto para professores de aerofotogeologia. SBG-CNPq, p. 173-209.

147 ELLERT, N. \& BARCHA, S. F. (1982) Aplicação de métodos geofísicos na delimitação do Alto Estrutural de Tanabi - NNW do Estado de São Paulo. Geociências, UNESP, 1: 75-82.

148 FAIRCHILD, T. R. (1982) Paleobotânica e palinologia na América do Sul. Bol. IG-USP, 13: 43-134. Coordenador Editorial.

149 GAMA Jr., E. G.; FÚLFARO, V. J.; BANDEIRA Jr., A. N.; FRANÇA, A. B.; SAAD, A. R.; SILVA, G. R.; SANTOS, M. V.; SIMON, F. M.; RÔLA, J. G. R.; CALDAS, A. F. F. D. (1982) Plano de avaliação da ocorrência de gás de Cuiabá Paulista. PAULIPETRO, Consórcio CESP/IPT, RT$129 / 82,78 \mathrm{p}$.

150 GUARDANI, R. \& VALARELLI, J. V. (1982) Termofosfato magnesiano potássico fundido. Concurso de inventor Governador do Estado de São Paulo de 1982. 30 p.

GUARDANI, R. \& VALARELLI, J. V. (1982) Processo de produção de fertilizante potássico e dióxido de enxofre. Patente de Invenção, IPT, Inst. Nac. Prop. Ind. no 8200560, 9p.

152 MARTIN, L.; MÖRNER, N. A.; FLEXOR, J. M.; SUGUIO, K. (1982) Reconstrução de antigos níveis marinhos do Quaternário. Publ. CTCQ/SBG/IGUSP, 154p.

153 MENDES, J. C. (1982) Paleontologia Geral, Rio de Janeiro, Livros Técnicos e Científicos Ed. S. A., 2a ed., 368p.

154 PETRI, S.; COIMBRA, A. M.; AMARAL, G.; OJEDA y OJEDA, H.A.; FÚlFARO, V. J.; PONÇANO, W. L. (1982) Código Brasileiro de Nomenclatura Estratigráfica e Guia de Nomenclatura Estratigráfica. Jornal do Geólogo, 17: 55p. (Suplemento especial).

REBOUÇAS, A. C. (1982) As Águas Subterrâneas na década mundial da água potável - Geologia CEPEGE, 8: 61-70.

RIBEIRO FILHO, E. (1982) The mineral Province of Carajás. Associação Nacional de Programação Econômica e Social, São Paulo, SP, 16p.

157 RIBEIRO FILHO, E. (1982) Anais do VI Simpósio Anual da Acadêmica de Ciências do Estado de São Paulo, vol. IV: 1-92. Coordenador Editorial de Geologia Ambiental.

158 RÖSLER, O. (1982) Coleções de megafósseis vegetais do Instituto de Geociências (Departamento de Paleontologia e Estratigrafia), Universidade de São Paulo. Bol. Ass. Latinoam. Paleobot. Palin., 8: $1-20$.

159 SUGUIO, K.; MEIS, M. R. M.; TESSLER, M. G. (1982) Atas do IV Simp. Quat. Brasil., Rio de Janeiro, RJ, Publ. Com. Técn. Cient. Quat., 482p. (Coordenadores Editoriais)

160 TASSINARI, C. C. G. (1982) Levantamento de Recursos Naturais, v. 26, Folha ao Milionésimo SD-21 Cuiabá. Projeto RADAMBRASIL, 9p.

161 TASSINARI, C. C. G. (1982) Levantamento de Recursos Naturais, v. 25, Folha ao Milionésimo SD22 Goiás. Projeto RADAMBRASIL, 58p.

162 TASSINARI, C. C. G. (1982) Levantamento de Recursos Naturais, v. 27, Folha ao Milionésimo, SE21 Corumbá, Projeto RADAMBRASIL, 20p.

163 TASSINARI, C. C. G. (1982) Comentários geocronológicos em rochas pré-cambrianas da Folha SC-23 Rio São Francisco-Salvador. Projeto RADAMBRASIL, Relatório Interno, 23p.

164 TEIXEIRA, W. (1982) Folha SH-22 Porto Alegre, SI-22 Lagoa Mirim e SH-21 Uruguaiana: interpretação dos dados radiométricos e evolução geocronológica. Projeto RADAMBRASIL, Relatório Interno, 35p.

165 TEIXEIRA, W. (1982) Folhas ao milionésimo SF-23 Rio de Janeiro e SF-24 Vitória: síntese interpretativa dos dados radiométricos. Projeto RADAMBRASIL, Relatório Interno, 47p. 
166 ARAI, M. \& RÖSLER, O. (1983) Megásporos de São João do Triunfo, Paraná, Formação Rio Bonito (Permiano). Paleobot. Latinoam., Circ. ALPP, 5 (1): 11.

167 BABINSKI, M. E. C. B. O. \& RÖSLER, O. (1983) Licófitas de Fluviopólis (Permiano Superior) no sul do Estado do Paraná. Paleobot. Latinoam., Circ. ALPP, 5: (1) : 6-7.

168 BATTAGIN, A. F. \& KIHARA, Y. (1983) Retrato mineralógico dos cimentos pozolânicos e de alto-forno produzidos no Brasil de 1980 a 1982. XXXIV Reunião de técnicos da indústria de cimento, ABCP, São Paulo, SP, Anexo, 1, 21p.

169 CAMPOS NETO, M. C.; BASEI, M. A. S.; ARTUR, A. C.; SILVA, M. E.; MACHADO, R.; DIAS NETO, C. M.; FRAGOSO CESAR, A. R.; SOUZA, A. P. (1983) Geologia das Folhas de Picaia e Igaratá. I Jornada sobre a Carta Geológica do Estado de São Paulo em 1: 50.000, PRÓ-MINÉRIO-IPT, p. 55-78.

170 CARVAlHo, A. \& MELFI, A. J. (1983) Lateritisation Processes - Proceedings of the II Inter. Semin. Laterit. Processes, São Paulo, SP, 590p. Coordenadores Editorais.

171 FRAGOSO CESAR, A. R. S. (1983) Evolução paleoambiental a tectônica da Bacia do Camaquã (RS): uma introdução. Dissertação de Mestrado, IG-UFRGS, Porto Alegre, 103p.

172 KIHARA, Y. (1983) Perfil analítico das cinzas volantes brasileiras. XXXIV Reunião de técnicos da indústria de cimento, ABCP, São Paulo, SP, Anexo 7, 30p.

173 LIMA, M. R. (1983) A aplicação geológica da palinologia. Geol. Ciênc. Técn., 9: 57-65.

174 MONTALVÃO, R. M. G. \& TASSINARI, C. C. G. (1983) Geocronologia dos granitóides e gnaisses da região do Rio Maria, Fazenda Mata Geral e Rio Itacaiúnas, sul do Pará, Projeto RADAMBRA. SIL, Relatório Interno, $8 \mathrm{p}$.

175 MUAMBA, K. \& REBOUÇAS, A. C. (1983) Carte des provinces hydrogeologiques - Zaire. Programme-ERTS, Zaire.

176 PERINOTTO, J. A. J. \& RÖSLER, O. (1983) Glossopterídeas de Fluviópolis (Permiano Superior) no sul do Estado do Paraná. Paleobot. Latinoam., Circ. ALPP, 5 (1): 8-9.

177 PETRI, S. \& FÚlfFARO, V. J.(1983) Geologia do Brasil - Fanerozóico. São Paulo, SP, Ed. Thomas Queiroz-EDUSP, 631p.

178 PETRI, S. \& MENDES, J. C. (1983) Brazil (cap.6) In: The Phanerozoic Geology of the World. II The Mesozoic. Amsterdam, Holland, Elsevier Sci. Publ., p. 151-179.

179 RIBEIRO FILHO, E. (1983) Em busca do conhecimento ecológico: uma introdução à metodologia. São Paulo, SP, Ed. Blücher Ltda, 110p. Autor de: "Geologia da Ilha do Cardoso e Exercícios de Geologia".

180 RIBEIRO FILHO, E.; BARBOUR, A. P.; MORESCHI, J. B.; SIGOLO, J. B. (1983) Projeto Fluoritaavaliação do potencial brasileiro de fluor. Relatório Interno, IG-USP, 116p.

181 RÖSLER, O. (1983) Introdução ao volume de Resumos da IV Reunião de Paleobotânicos e Palinólogos, São Paulo, SP, Paleobot. Latinoam., Circ. ALPP, 5 (1): 2.

182 ROSLER, O \& FITIPPALDI, F. C. (1983) Os estudos cuticulares em plantas fósseis: seu desenvolvimento e suas perspectivas. Paleobot. Latinoam., Circ., ALPP, 5 (1): 5.

183 ROSLER, O. \& ROHN, R. (1983) Sphenophyllum paranaensis n. sp. (Sphenophyta) da Formação do Rio do Rastro (Permiano Superior) de Dorizon, Estado do Paraná, IV Reunião de Paleobotânicos e Palinólogos, São Paulo, SP, Paleobot. Latinoam., Circ. ALPP, 5 (1): 10.

184 VALARELLI, J. V. \& GUARDANI, R. (1983) Processo de produção de fertilizantes potássicos e dióxido de enxofre. Concurso de inventor Governador do Estado de São Paulo de 1983. 34p. 